\title{
Aplicación del método PÓLYA para mejorar el rendimiento académico de matemática en los estudiantes de secundaria
}

\section{Application of the PÓLYA method to improve the academic performance of mathematics in high school students}

\author{
Dr. José Luis Rodríguez López \\ Dra. Judith Soledad Yangali Vicente \\ Universidad César Vallejo, Perú \\ Autor para correspondencia: judithsyv@ gmail.com \\ Fecha de recepción: 30 de Agosto de 2016 - Fecha de aceptación: 25 de Octubre de 2016
}

Resumen: El presente estudio tuvo por finalidad determinar la aplicación del Método de Pólya, para mejorar el rendimiento académico en matemática de los estudiantes del quinto grado de educación secundaria. El método de Pólya consiste en una secuencia de pasos que van desde la comprensión del problema hasta la evaluación de los procedimientos empleados en la resolución de un problema matemático. Entiéndase como rendimiento académico a la medida de las capacidades del estudiante que expresa lo que este ha aprendido a lo largo de un proceso formativo. En cuanto a la metodología, los datos fueron obtenidos mediante la aplicación de test registrando los resultados en una guía de observación, instrumento que fue evaluado por el criterio de expertos confirmando su validez en cuanto a su construcción y contenido. Luego se procedió al tratamiento de dicha información mediante el programa estadístico SPSS en una población de 120 estudiantes, 60 del grupo experimental y 60 del grupo control. Los resultados arrojados en este estudio fueron muy satisfactorios tanto para los estudiantes como para los docentes en el nivel de logro, del Rendimiento Académico en matemáticas, puesto que la media de calificaciones pos-test fue de 15,89 frente al 10,72 del pre-test, evidenciándose un incremento del 67,46\%, alcanzando el nivel de logro previsto por parte de los estudiantes. Los resultados obtenidos apuntan pues a que la aplicación del Método de Pólya en la resolución de problemas mejora significativamente el rendimiento académico en matemática de los estudiantes de educación secundaria.

Palabras claves: dinero electrónico; incentivo tributario; medio de pago

Abstract: This study aimed to determine the application of the method Pólya, to improve academic performance in mathematics of fifth grade students of secondary education. Polya method consists of a sequence of steps ranging from understanding the problem to evaluating the procedures used in solving a mathematical problem. Understood as academic performance to measure a student's abilities that expresses what it has learned during a training process. In terms of methodology, data were obtained by applying recording test results in an observation guide, instrument was evaluated by expert judgment confirming the validity as to its construction and content. Then he proceeded to the processing of such information through the SPSS statistical program in a population of 120 students, 60 in the experimental group and 60 in the control group. The results obtained in this study were very satisfactory for both students and teachers in the level of achievement, the Academic Performance in mathematics, since the average post-test scores was 15.89 against 10.72 the pre -test, showing an increase of $67.46 \%$, reaching the level of achievement expected by 
students. The results suggest that the application for Method Polya in solving problems significantly improves academic performance in mathematics of students in secondary education.

Key words: polya method; academic; achievement

\section{Introducción}

Uno de los más preciados patrimonios de la humanidad es sin lugar a dudas las matemáticas. Tal como lo manifestó Pineda (2009), "las maravillas tecnológicas y el avance vertiginoso de las mismas se deben, en gran parte, al avance y a la investigación que se desarrolla en matemáticas a lo largo de todo el mundo" (p.47). Las dificultades en el proceso de enseñanza y aprendizaje de la matemática en los estudiantes de los diferentes niveles de la educación básica y superior han sido motivo tanto para investigadores, como para los docentes la búsqueda constante de nuevas metodologías y herramientas que permitan lograr el desarrollo de las capacidades matemáticas y por ende elevar el rendimiento académico de los estudiantes. Este estudio tiene como finalidad proporcionar una alternativa, en la forma de enseñar y aprender matemática; la aplicación de un método de resolución de problemas como el método de Pólya cuyos pasos seguidos en forma ordenada y secuencial permitirá la resolución óptima de los problemas matemáticos, mejorando así el rendimiento académico. Los diferentes informes de educación internacionales indican que el rendimiento escolar en el área de matemática se encuentra por debajo de lo esperado y deseado.

El Perú no es ajeno a esto, pues la matemática es una de las disciplinas que mayor problema presenta en cuánto a rendimiento académico se refiere. El insuficiente nivel académico de los estudiantes se ha convertido en uno de los grandes problemas que afronta en la actualidad el sistema educativo de nuestra sociedad. La nota promedio que establece la Organización para la Cooperación y el Desarrollo Económico (OCDE) para el rubro de matemática del Programa para la Evaluación Internacional de Estudiantes (PISA) es de 494. Sin embargo, Perú no solo obtuvo un puntaje muy lejano a este promedio, sino que ocupó el último lugar en esta categoría. 368, fue la nota que obtuvo, siendo superada por los otros 64 países participantes de la evaluación. Perú también ha participado en las evaluaciones del 2001 y el 2009. En esta última prueba, ocupamos el penúltimo lugar en ciencia y el antepenúltimo lugar en matemática y comprensión lectora. Diversos indicadores expresan que los estudiantes no logran los aprendizajes esperados por qué no desarrollan adecuadamente las capacidades matemáticas. Ello se evidenció en las diferentes evaluaciones que se aplicaron para verificar el aprendizaje de los estudiantes del Colegio Militar Leoncio Prado (CMLP) al hacer el análisis de las calificaciones de la unidad de aprendizaje inmediata anterior.

\section{Problema}

¿En qué medida la aplicación del Método de Pólya en la resolución de problemas permiten mejorar el rendimiento académico en matemática de los estudiantes de quinto grado de secundaria del Colegio Militar Leoncio Prado de La Perla, Callao?

\section{Objetivo}


Determinar la aplicación del Método de Pólya en la resolución de problemas para mejorar significativamente el rendimiento académico en matemática de los estudiantes de quinto grado de secundaria del Colegio Militar Leoncio Prado de La Perla, Callao.

\section{Hipótesis}

La aplicación del Método de Pólya en la resolución de problemas mejora significativamente el rendimiento académico en matemática de los estudiantes de quinto grado de secundaria del Colegio Militar Leoncio Prado de La Perla, Callao.

\section{Justificación}

Consideramos esta investigación pertinente y relevante dado que el aprendizaje de la matemática es fundamental, pues está presente en los diversos aspectos de la actividad humana, actividades en el ámbito social, familiar, cultural y en la naturaleza misma. El rendimiento académico en el área de matemática en nuestro país es preocupante, pues durante el proceso de aprendizaje los estudiantes no han logrado desarrollar satisfactoriamente las capacidades matemáticas porque la forma como se les ha enseñado no ha despertado su interés por el curso y por el contrario las han encontrado aburridas, complicadas inútiles y sin relación con su vida cotidiana. Esto debido a que se ha enseñado de la forma tradicional. Este nuevo enfoque de resolución de problemas orienta la actividad matemática en la escuela, pues propone que al estudiante se le debe situar en diversos contextos con la finalidad de crear, recrear, investigar y resolver problemas (MINEDU, Rutas del aprendizaje, 2015). El método Pólya es una estrategia novedosa que servirá de herramienta en el proceso de enseñanza de la matemática además de dar la oportunidad de desarrollar el potencial constructivo de los estudiantes. Por tal razón, se propone la aplicación del método de Pólya como estrategia didáctica en el proceso de enseñanza de la matemática, pues pensamos que su aplicación mejorará el rendimiento académico de los estudiantes en ésta área. Por lo cual el siguiente estudio se justifica de la siguiente manera: Constituye un aporte a la cultura pedagógica, pues dará a conocer un nuevo enfoque en la enseñanza y aprendizaje de la matemática que pretende conseguir el desarrollo del pensamiento matemático y, en consecuencia, el desarrollo de las capacidades y competencias matemáticas.

Según Guskey (2013) el rendimiento académico del estudiante es un constructo multifacético, que está relacionado con diferentes dominios de aprendizaje, que se mide de formas distintas y con diferentes propósitos (p. 125). Asimismo partiendo de la definición de Jiménez (2000), la cual postula que: El rendimiento escolar es un "nivel de conocimientos demostrado en un área o materia comparado con la norma de edad y nivel académico (p. 198). Por tanto, el rendimiento del alumno debería ser entendido a partir de sus procesos de evaluación, sin embargo. La simple medición y/o evaluación de los rendimientos alcanzados por los alumnos no provee por sí misma todas las pautas necesarias para la acción destinada al mejoramiento de la calidad educativa. En el mejor de los casos, si pretendemos conceptualizar el rendimiento académico a partir de su evaluación, es necesario considerar no solamente el desempeño individual del estudiante sino la manera como es influido por el grupo de pares, el aula o el propio contexto educativo. Probablemente una de las variables más empleadas o consideradas por los docentes e investigadores para aproximarse al rendimiento académico son: las calificaciones escolares ; razón de ello que existan estudios que pretendan calcular algunos 
índices de fiabilidad y validez de éste criterio considerado como 'predictivo' del rendimiento académico (no alcanzamos una puesta en común de su definición y sin embargo pretendemos predecirlo), aunque en la realidad del aula, el investigador incipiente podría anticipar sin complicaciones, teóricas o metodológicas, los alcances de predecir la dimensión cualitativa del rendimiento académico a partir de datos cuantitativos.

Dimensiones del rendimiento académico. Las dimensiones del Rendimiento Académico en matemáticas son las capacidades Matemáticas. El Ministerio de Educación (2015) en su fascículo de rutas de aprendizaje, señaló: Las Capacidades matemáticas son facultades de toda persona para actuar conscientemente sobre la realidad, sea para resolver un problema o cumplir un objetivo, haciendo uso flexible y creativo de los conocimientos, las habilidades, las destrezas, la información o las herramientas que tenga disponibles y considere pertinentes a la situación. La resolución de situaciones problemáticas es una competencia matemática importante que nos permite desarrollar capacidades matemáticas. Todas ellas existen de manera integrada y única en cada persona y se desarrollan en el aula, la escuela, la comunidad, en la medida que dispongamos de oportunidades y medios para hacerlo. En otras palabras, las capacidades matemáticas se despliegan a partir de las experiencias y expectativas de nuestros estudiantes, en situaciones problemáticas reales. Si ellos encuentran útil en su vida diaria los aprendizajes logrados, sentirán que la matemática tiene sentido y pertinencia. La propuesta pedagógica para el aprendizaje de la matemática toma en cuenta el desarrollo de cuatro capacidades matemáticas, consideradas esenciales para el uso de la matemática en la vida cotidiana. Éstas sustentan las competencias matemáticas y deben abordarse en todos los niveles y modalidades de la Educación Básica Regular. Estas cuatro capacidades matemáticas serán consideradas como dimensiones y son las siguientes:

Dimensión 1: Matematiza situaciones. Es la capacidad de expresar un problema, reconocido en una situación, en un modelo matemático. En su desarrollo se usa, interpreta y evalúa el modelo matemático, de acuerdo a la situación que le dio origen.

Dimensión 2: Comunica y representa ideas matemáticas. Es la capacidad de comprender el significado de las ideas matemáticas, y expresarlas en forma oral y escrita usando el lenguaje matemático y diversas formas de representación con material concreto, gráfico, tablas, símbolos y recursos TIC, y transitando de una representación a otra.

Dimensión 3: Elabora y usa estrategias. Es la capacidad de planificar, ejecutar y valorar una secuencia organizada de estrategias y diversos recursos, entre ellos las tecnologías de información y comunicación, empleándolas de manera flexible y eficaz en el planteamiento y resolución de problemas, incluidos los matemáticos. Esto implica ser capaz de elaborar un plan de solución, monitorear su ejecución, pudiendo incluso reformular el plan en el mismo proceso con la finalidad de llegar a la meta. Asimismo, revisar todo el proceso de resolución, reconociendo si las estrategias y herramientas fueron usadas de manera apropiada y óptima.

Dimensión 4: Razona y argumenta generando ideas matemáticas. Es la capacidad de plantear supuestos, conjeturas e hipótesis de implicancia matemática mediante diversas formas de razonamiento (deductivo, inductivo y abductivo), así como el verificarlos y validarlos usando argumentos. Esto implica partir de la exploración de situaciones vinculadas a la matemática para 
establecer relaciones entre ideas, establecer conclusiones a partir de inferencias y deducciones que permitan generar nuevas conexiones e ideas matemáticas.

Por tanto se justifica la presente investigación porque sirvió de apoyo al docente pues le dio una herramienta pedagógica que le permitirá desarrollar sus sesiones de aprendizaje de una manera más didáctica mediante la aplicación del método de Pólya en la resolución de problemas. Lo esencial en la enseñanza de la matemática es desarrollar tácticas en la resolución de problemas y el Método de Pólya permitirá a los estudiantes aplicar una serie de procedimientos que en realidad se utilizan y aplican en cualquier campo de la vida diaria.

\section{Método}

La investigación es de tipo aplicada, de carácter exploratorio, de diseño experimental, nivel cuasi-experimental, según su naturaleza es cuantitativa, según su alcance es longitudinal. El método es experimental; según Sánchez \& reyes (2002) el método experimental consiste en organizar deliberadamente condiciones de acuerdo con un plan previo con el fin de investigar las posibles relaciones causa-efecto exponiendo a uno o varios grupos experimentales a la acción de una variable experimental y contrastando sus resultados con grupos de control o comparación (p. 43). La población objeto de estudio, estuvo constituida por 120 estudiantes, 60 estudiantes conformaron el grupo de control y 60 estudiantes el grupo experimental correspondiente a dos secciones cada una. El método de muestreo fue no probabilístico, este se refiere a una porción de la población en el que la elección de los elementos no depende de la probabilidad sino de las características y criterios de la investigación según la definición de Hernández, Fernández y Baptista (2010). Por tanto, la selección de los grupos fue a criterio del investigador.

\section{El instrumento}

En la investigación se utilizó una guía de observación con los indicadores de evaluación respectivos, donde se registraron las calificaciones obtenidas en las fichas de trabajo y evaluación aplicada en el proceso de aprendizaje.

\section{Confiablidad}

La validación del instrumento se realizó mediante el criterio o juicio de expertos. La confiabilidad se determinó mediante la aplicación del método estadístico de Kuder-Richardson$20(\mathrm{Kr}-20)$ arrojando un nivel de confiabilidad de 0,61.

\section{Método de análisis de Datos}

Una vez recolectada la información obtenida mediante la guía de observación se procedió a la elaboración de la base de datos y su respectivo procesamiento. Para ello se sistematizo la información de acuerdo a los criterios que fueron formulados expresamente para este estudio. Este análisis se dio en las siguientes etapas: ordenamiento, procesamiento (elaboración de cuadros y gráficos estadísticos), consolidación y presentación de los datos recogidos con el instrumento. En el análisis estadístico se utilizaron indicadores como la media, mediana, moda, 
desviación estándar y varianza; con un nivel de confianza alto, haciendo uso de los programas Excel y SPSS versión 22.

\section{Resultados}

La hipótesis en la investigación fue determinar si la aplicación del Método de Pólya en la resolución de problemas mejora significativamente el rendimiento académico en matemática de los estudiantes de quinto grado de secundaria del Colegio Militar Leoncio Prado de La Perla, Callao. Luego de aplicar el Método en el proceso de aprendizaje se realizaron los análisis de los resultados obtenidos mediante la aplicación de la Prueba T para muestras independientes a fin de verificar si efectivamente aceptábamos o no la hipótesis de la investigación.

Tabla 1.

Comparación de medias de los grupos Pre test

\begin{tabular}{|c|c|c|c|c|c|}
\hline \multicolumn{6}{|c|}{ Estadísticas de grupo } \\
\hline & Grupo & $\mathrm{N}$ & Media & $\begin{array}{l}\text { Desviación } \\
\text { estándar }\end{array}$ & $\begin{array}{l}\text { Media de error } \\
\text { estándar }\end{array}$ \\
\hline \multirow[t]{2}{*}{ Matematiza situaciones } & control & 60 & 10,15 & 1,830 & ,236 \\
\hline & experimental & 60 & 10,82 & 1,944 & ,251 \\
\hline \multirow{2}{*}{$\begin{array}{l}\text { Comunica y representa } \\
\text { ideas matemáticas }\end{array}$} & control & 60 & 11,00 & 1,756 & ,227 \\
\hline & experimental & 60 & 11,05 & 1,854 & 239 \\
\hline \multirow[t]{2}{*}{ Elabora y usa estrategias } & control & 60 & 10,97 & 1,922 & ,248 \\
\hline & experimental & 60 & 10,95 & 1,780 & 230 \\
\hline \multirow{2}{*}{$\begin{array}{c}\text { Razona y argumenta ideas } \\
\text { matemáticas }\end{array}$} & control & 60 & 10,72 & 1,698 & ,219 \\
\hline & experimental & 60 & 10,08 & 1,844 & ,238 \\
\hline
\end{tabular}

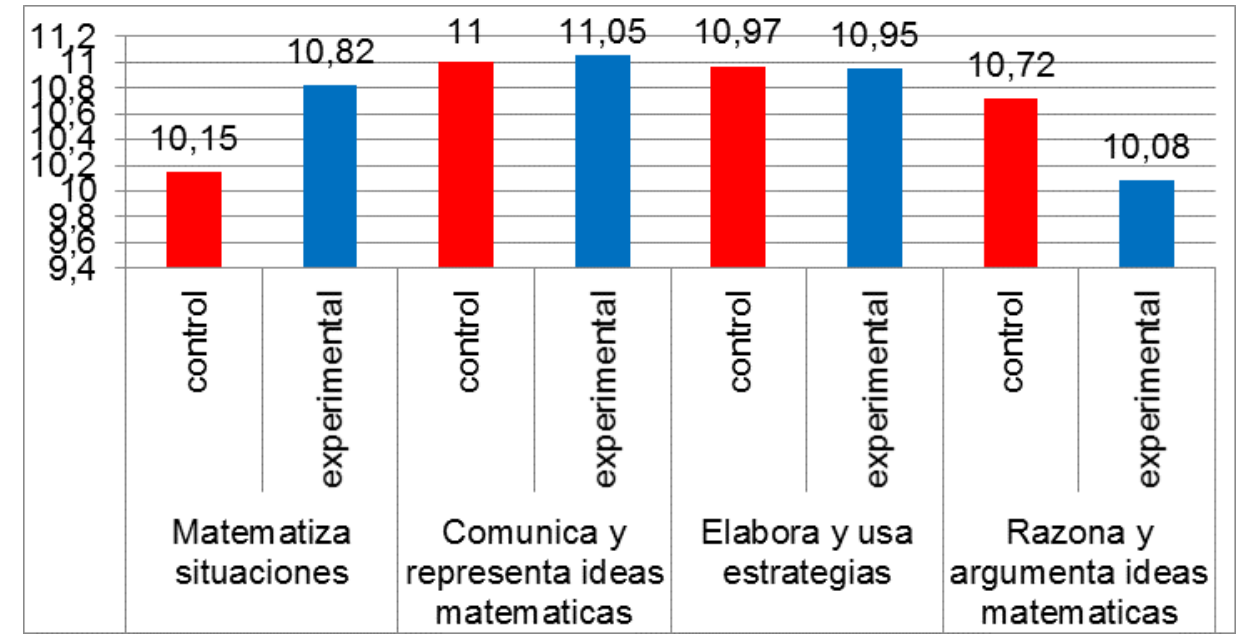

Figura 1. Media calificaciones pre test.

En la tabla 1 y figura 1 observamos la diferencia entre las medias de las calificaciones en las capacidades matemáticas de cada grupo antes de aplicar el programa del geogebra con el método de Pólya.

Tabla 2.

Comparación de medias de los grupos Pos test 


\begin{tabular}{clcccc}
\multicolumn{1}{c}{ Dimensiones } & \multicolumn{1}{c}{ Grupo } & $\mathrm{N}$ & Media & $\begin{array}{c}\text { Desviación } \\
\text { estándar }\end{array}$ & $\begin{array}{c}\text { Media de error } \\
\text { estándar }\end{array}$ \\
\hline Matematiza situaciones & Control & 60 & 10,97 & 1,886 &, 244 \\
& Experimental & 60 & 16,02 & 1,790 &, 231 \\
Comunica y representa ideas & Control & 60 & 10,82 & 1,780 &, 230 \\
matemáticas & Experimental & 60 & 15,97 & 1,850 &, 239 \\
Elabora y usa estrategias & Control & 60 & 10,08 & 1,816 &, 234 \\
& Experimental & 60 & 15,35 & 1,876 &, 242 \\
Razona y argumenta ideas & Control & 60 & 11,03 & 1,775 &, 229 \\
matemáticas & Experimental & 60 & 16,22 & 1,833 &, 237 \\
\hline
\end{tabular}

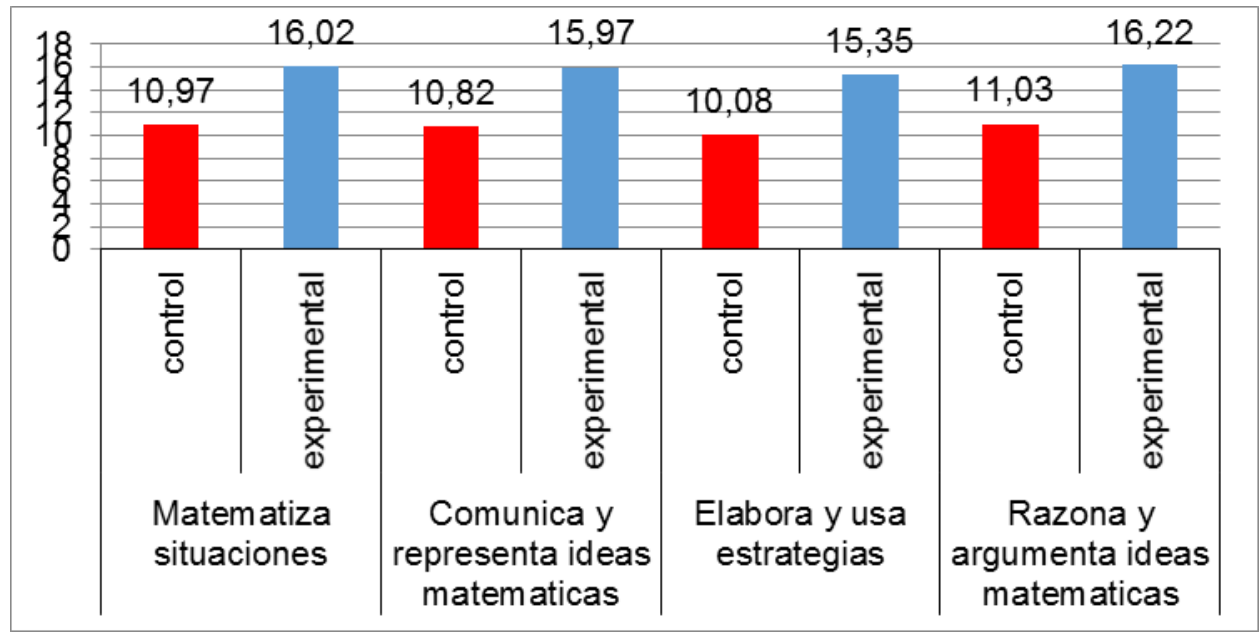

Figura 2. Media calificaciones Pos test

En la tabla 2 y figura 2 observamos las diferencias entre las medias de las calificaciones en las capacidades matemáticas de cada grupo después de aplicar el programa del geogebra con el método de Pólya.

Tabla 3.

Registro de evaluaciones del rendimiento académico Pos test

\begin{tabular}{lccccc}
\hline Grupo & \multicolumn{4}{c}{ Capacidad } & Media \\
\hline & $\begin{array}{c}\text { Matematiza } \\
\text { situaciones }\end{array}$ & $\begin{array}{c}\text { Comunica y } \\
\text { representa ideas } \\
\text { matemáticas }\end{array}$ & $\begin{array}{c}\text { Elabora y } \\
\text { usa } \\
\text { estrategias }\end{array}$ & $\begin{array}{c}\text { Razona y } \\
\text { argumenta ideas } \\
\text { matemáticas }\end{array}$ & \\
Control & 10,97 & 10,82 & 10,08 & 11,03 & 10,725 \\
Experimental & 16,02 & 15,97 & 15,35 & 16,22 & 15,89 \\
\hline
\end{tabular}

En la tabla 3 se observa las medias de cada capacidad de cada grupo así como también el promedia del rendimiento académico total después del pos test.

\section{Conclusión}

Luego del análisis estadístico respectivo observamos una diferencia significativa entre las medias de las calificaciones de los grupos experimental y de control en el pre-test y pos-test respectivamente puesto que el valor de $\mathrm{p}=0,00$ está por debajo del $\alpha=0,05$ por lo que se concluye que la aplicación del Método de Pólya en la resolución de problemas mejora 
significativamente el rendimiento académico en matemática de los estudiantes de quinto grado de secundaria del Colegio Militar Leoncio Prado de La Perla, Callao. Como consecuencia de la aceptación de las hipótesis específicas alternativas; ya que hubo un aumento del 48,16\% entre las medias de las calificaciones en el grupo experimental mientras que en el grupo control no se evidenció diferencia alguna en el pre-test y el pos-test respectivamente a un nivel de confianza de $95 \%$.

\section{Agradecimientos}

A la Universidad César Vallejo y a los docentes del Doctorado en Educación de la sede La Autónoma, por las oportunidades de desarrollo profesional.

A nuestro Asesor Dr. Jaime Agustín Sánchez Ortega, por su apoyo profesional y transmisión de oportunidades de crecimiento profesional, en la carrera.

A nuestras familias, a los amigos(as) y compañeros del Doctorado en Educación de la sede Autónoma, por el apoyo, por la experiencia vivida, por los buenos momentos y sobre todo por la amistad.

\section{Bibliografía}

Borgonovo, D. (2013). Deseo de saber y rendimiento académico. Puntos de encuentros. Argentina: UFASTA.

Boscán, M., y Klever, K. (2012). Metodología basada en el método heurístico de Polya para el aprendizaje de la resolución de problemas matemáticos . Escenarios.

Castejón, J. L. (2014). Aprendizaje y Rendimiento Académico. Alicante. Editora Club Universitario.

Echeverría, C. (2012). Fundesa. Obtenido de http://www.fundesa.org.gt/cms/content/files/Comunicado_de_Prensa_\%C3\%8Dndice_Gl obal_TICs_2012(1).pdf

Echeverría, P. (2010). Rendimiendo académico en Matemática. Guatemala: Tesis inédita. Universidad de San Carlos de Guatemala.

Firgermann. (2010). Calidad de aprendizaje. La guía educativa. Obtenido de http://educacion.laguia2000.com/aprendizaje/calidad-del-aprendizaje

Ministerio de Educación . (2015). Diseño Curricular Nacional. Lima: MINEDU.

Peña, K. (2008). Método de Polya en el diseño de estrategias para facilitar la resolución de problemas relacionados con áreas de figuras planas. Venezuela: UNV.

Peña, K. (2008). Método de Polya en el diseño de estrategias para facilitar la resolución de problemas relacionados con áreas de figuras planas. Venezuela: UNV. 
Prieto . (2015). Característica de la calidad d apre Peña, K. (2008). Método de Polya en el diseño de estrategias para facilitar la resolución de problemas relacionados con áreas de figuras planas. Venezuela: UNV.

Rodríguez . (2013). Factores para mejorar la calidad del aprendizaje del estudiante universitario. Lima, Perú: Revistas de investigación UNMSM. Obtenido de http://revistasinvestigacion.unmsm.edu.pe/index.php/educa/article/view/7690

Roque, J. W., y Contreras , P. C. (2009). Influencia de la enseñanza de la matemática basada en la resolución de problemas en el mejoramiento del rendimiento académico. Universidad Nacional Mayor de San Marcos. Programa Cybertesis PERÚ. Obtenido de http://cybertesis.unmsm.edu.pe/handle/cybertesis/1704 